\title{
A VIDAV-PALMER THEOREM FOR JORDAN C*-ALGEBRAS AND RELATED TOPICS
}

\author{
ANGEL RODRÍGUEZ PALACIOS
}

\section{Introduction}

The Vidav-Palmer theorem asserts that for a unital Banach (associative) complex algebra $A$ to admit an involution which turns it into a $C^{*}$-algebra, it is a necessary and sufficient condition that $A$ should be a $\mathrm{V}$-algebra, and then the involution is determined by the formula:

$$
h+i k \stackrel{*}{\longrightarrow} h-i k \quad(h, k \in H(A))
$$

the "natural involution of the V-algebra $A$ " (for the concept of V-algebra and the definition of $H(A)$ see Section 1 of this paper). In view of this theorem, nonassociative $\mathrm{V}$-algebras are an available model for nonassociative $\mathrm{C}^{*}$-algebras.

The above mentioned theorem of Vidav and Palmer consists of two assertions with independent proofs:

(a) $(a b)^{*}=b^{*} a^{*}(a, b \in A)$, "multiplicativity of the natural involution of the associative V-algebra $A$ ".

(b) $\left\|a^{*} a\right\|=\|a\|^{2}(a \in A)$, "star-property of the natural involution".

A result of J. Martinez, A. Mojtar and the author (see [10]) proves that the natural involution of a nonassociative $\mathrm{V}$-algebra $A$ is multiplicative if and only if $A$ is a noncommutative Jordan algebra (thus determining the widest context in which assertion (a) is valid in the nonassociative case). So noncommutative Jordan Valgebras are a good generalization of associative $C^{*}$-algebras (note in addition their good algebraic behaviour: power-associativity, existence of an available concept of inverse for some of their elements, etc.). The purpose of this paper is to continue the study of noncommutative Jordan V-algebras begun in [10].

The basic tool is the recent theorem of the Vidav-Palmer type due to J. Martinez [8] and M. A. Youngson ([19] and [20]) showing that the class of (commutative) Jordan V-algebras is just the one of Jordan $C^{*}$-algebras. The concept of Jordan $C^{*}$ algebra has been formulated by Kaplansky using axioms which we can call "of the Gelfand-Naimark type" and it has been studied by several authors since the work of Wright [15] whose main result establishes that real JB-algebras in the sense of Alfsen, Schultz and Störmer [1] are just the selfadjoint parts of Jordan $C^{*}$-algebras.

In Section 1 of this paper I give a new proof of the above-mentioned result by Martinez and Youngson (Theorem 3) and from this and the papers [10], [15] and [17] we obtain interesting consequences in the theory of V-algebras, especially of noncommutative Jordan V-algebras (Theorems 8 and 10) which are useful in the following sections.

Received 27 February, 1979; revised 30 October, 1979.

[J. LONDON MATH. SOC. (2), 22 (1980), 318-332] 
Sections 2, 3 and 4 are independent (only Corollary 25 needs Theorem 14).

In Section 2 unital nonassociative $\mathrm{B}^{*}$-algebras are defined (by requiring exactly the Gelfand-Naimark axiom) and it is shown (Theorem 14) that such a $\mathrm{B}^{*}$-algebra is always alternative. Since the multiplicativity of the involution is not assumed, this result includes in particular a complete nonassociative parallel to assertion (b) in the Vidav-Palmer theorem: the natural involution of a nonassociative V-algebra satisfies the star-property if and only if the algebra is alternative (see also Corollary 9). As a consequence (curiously), assertion (b) of the Vidav-Palmer theorem implies (a).

In Section 3 the bidual of a noncommutative Jordan V-algebra with the Arens product is studied and it is shown (Theorem 23) that this algebra is a new noncommutative Jordan V-algebra satisfying every multilinear identity satisfied by the first. In consequence, the bidual of a Jordan $C^{*}$-algebra is another Jordan $C^{*}$ algebra (Corollary 24).

Section 4 is devoted to proving that the algebraic numerical index (Theorem 26) and the normed space numerical index (Corollary 33) of a noncommutative Jordan $\mathrm{V}$-algebra is either 1 or $\frac{1}{2}$ depending on whether or not $A$ is associative and commutative.

\section{Previous concepts and results}

Two terminologies are used in this paper; the first is the one of nonassociative algebras, for which we refer to [13] in the general case and to [6] in the particular case of Jordan algebras, and the second is the one of numerical ranges in nonassociative unital normed algebras.

A unital normed algebra will be a nonassociative real or complex algebra $A$ with identity $I$ in which a norm is defined, satisfying:

$$
\|a b\| \leqslant\|a\|\|b\| \quad(a, b \in A), \quad\|I\|=1 .
$$

If $a$ is an element of such an algebra $A$, we define the numerical range of $a$ $(V(A, a)$ or $V(a)$ when confusion is not possible) by

$$
V(A, a)=\left\{f(a): f \in A^{\prime},\|f\|=f(I)=1\right\}
$$

which is a nonempty compact convex subset of the scalar field $\mathbb{R}$ or $\mathbb{C}$. The numerical radius of $a(v(a))$ is defined by

$$
v(a)=\sup \{|c|: c \in V(a)\} .
$$

Our first basic result is easily verified.

Proposition 1. If $A$ and $B$ are unital normed algebras over the same field and $F$ is a continuous linear mapping from $A$ into $B$ with norm one (respectively isometric) and such that $F(I)=I$, then $V(B, F(a)) \subset V(A, a)$ (respectively $V(B, F(a))=V(A, a)$ ) for all $a \in A$.

For each element $a$ of an algebra $A$, we shall denote by $L_{a}$ and $R_{a}$ the mappings $b \rightarrow a b$ and $b \rightarrow b a$ from $A$ into $A$. Since $A$ is a unital normed algebra we have

$$
\left\|L_{a}\right\|=\left\|R_{a}\right\|=\|a\| \quad(a \in A), \quad L_{I}=R_{I}=I
$$


and denoting by $B L(A)$ the (unital normed associative) algebra of bounded linear operators on $A$, we have the following corollary.

Corollary 2. For an element a of a unital normed algebra $A$

(a) $V(A, a)=V\left(B L(A), L_{a}\right)=V\left(B L(A), R_{a}\right)$,

(b) if $B$ is a subalgebra of $A$ with $\{a, I\} \subset B$, then $V(B, a)=V(A, a)$.

As the author does not know a systematic and easily accessible reference for numerical ranges in nonassociative unital normed algebras, the previous corollary becomes a basic tool in order to state in the present case some results well known for the associative case which can be found in [3] and [4] (perhaps it should be observed that $B L(A)$ is always associative and that the subalgebra $B$ can be chosen associative and closed if $A$ is power-associative).

Definitions. An element $a$ of a unital normed complex algebra $A$ will be called hermitian if $V(a) \subset \mathbb{R}$. The set of hermitian elements of $A$ will be denoted by $H(A)$.

A V-algebra will be a unital complete normed complex algebra $A$ satisfying $A=H(A)+i H(A)$ (the sum is automatically topological-direct).

An involution * in a complex vector space $X$ is a mapping from $X$ into $X$ satisfying

$$
(x+y)^{*}=x^{*}+y^{*},(c x)^{*}=\bar{c} x^{*},\left(x^{*}\right)^{*}=x(x, y \in X ; c \in \mathbb{C}) .
$$

An involution ${ }^{*}$ in a complex algebra $A$ will be called multiplicative if $(a b)^{*}=b^{*} a^{*}(a, b \in A)$.

If $A$ is a $V$-algebra, the mapping

$$
h+i k \longrightarrow h-i k \quad(h, k \in H(A))
$$

is a continuous involution on $A$ which we call "natural".

A Jordan $C^{*}$-algebra in the sense of Kaplansky is a unital complete normed complex Jordan algebra $A$ with a multiplicative involution * satisfying

$$
\left\|U_{a}\left(a^{*}\right)\right\|=\|a\|^{3} \quad(a \in A)
$$

(where $U_{a}=2 L_{a}^{2}-L_{a^{2}}$ ) .

Every Jordan $\mathrm{C}^{*}$-algebra is a Jordan $\mathrm{V}$-algebra whose natural involution is the previous one (see [19; Theorem 7]). Conversely, in [20; Theorem 8] it is shown that every Jordan $\mathrm{V}$-algebra with its natural involution is a Jordan $\mathrm{C}^{*}$-algebra, assuming that this involution is multiplicative. But this assumption is superfluous (see [8; Theorem IV.5.3]). So a theorem of the Vidav-Palmer type is true for Jordan C*algebras. In this section, using the above-mentioned result of [8], we give a new proof of this theorem and we obtain some consequences. To this end we need some auxiliary results which have their own interest.

If $X$ is a nonempty set and $A$ is a unital normed algebra, then the set of bounded functions from $X$ into $A$ is, with pointwise operations and supremum norm, a new unital normed algebra denoted $B(X, A)$. 
Proposition 3. If $A$ is complete and power-associative and $F \in B(X, A)$, then

$$
V(B(X, A), F)=\overline{\operatorname{co}} \bigcup\{V(A, F(x)): x \in X\}
$$

(co denotes closed convex hull).

Proof. By [3; Theorem 3.4] we have

$$
\begin{aligned}
\sup \operatorname{Re} V(F) & =\sup \left\{\frac{1}{r} \log \|\exp (r F)\|: r>0\right\} \\
& =\sup \left\{\sup \left\{\frac{1}{r} \log \|\exp (r F)(x)\|: x \in X\right\}: r>0\right\} \\
& =\sup \{\sup \operatorname{Re} V(F(x)): x \in X\} \\
& =\sup \operatorname{Re} \bigcup\{V(F(x)): x \in X\} \\
& =\sup \operatorname{Re}(\overline{\operatorname{co}} \bigcup\{V(F(x)): x \in X\}) .
\end{aligned}
$$

Remark 4. The assertion in Proposition 3 may be obtained without completeness (use the algebra completion of $A$ and apply Corollary 2) and without power associativity, for the crucial "exponential formula", which through Corollary 2 we have applied here, may be obtained in general nonassociative case.

Theorem 5. Let $A$ be a Jordan $\mathrm{C}^{*}$-algebra and let $T \in B L(A)$. Then

$$
V(B L(A), T)=\overline{\operatorname{co}} \bigcup\left\{V\left(A, \exp \left(-i L_{a}\right) T(\exp (i a))\right): a \in \operatorname{Sym}(A)\right\}
$$

$\left(\operatorname{Sym}(A)=\left\{a \in A: a^{*}=a\right\}\right)$.

Proof. For every $T \in B L(A)$ let $\hat{T}$ be the function from $\operatorname{Sym}(A)$ into $A$ defined by the formula

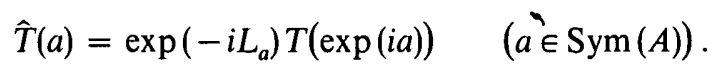

Let $a \in \operatorname{Sym}(A)$. By [19; Theorem 7], $a \in H(A)$ and hence $L_{a} \in H(B L(A))$ in view of Corollary 2(a). Applying [3; Lemma 5.2], $\exp \left(-i L_{a}\right)$ is isometric and so the equality $\|\hat{T}(a)\|=\|T(\exp (i a))\|$ implies

$$
\|\hat{T}\|=\sup \{\|\hat{T}(a)\|: a \in \operatorname{Sym}(A)\}=\sup \{\|T(\exp (i a))\|: a \in \operatorname{Sym}(A)\}=\|T\|
$$

(the last equality follows from [16; Corollary 2.4]).

The mapping $T \rightarrow \hat{T}$ is thus a unit preserving linear isometric mapping from $B L(A)$ into $B(\operatorname{Sym}(A), A)$. Applying consecutively Propositions 1 and 3 the result follows.

We come now to the promised new proof of the Vidav-Palmer theorem for Jordan $C^{*}$-algebras ([8] and [20]). 
Theorem 6. Let $A$ be a Jordan V-algebra. Then $A$ with its natural involution is a Jordan $\mathrm{C}^{*}$-algebra.

Proof. Let ${ }^{*}$ be the natural involution of $A$, which is continuous. By [8; Theorems IV.5.3 and IV.5.16], * is multiplicative and *-Sym $(A)(=H(A))$ is a JBalgebra in the sense of [1]. Applying [15; Theorem 2.8] we have an equivalent norm | $\mid$ on $A$ such that $\left(A,{ }^{*},|\cdot|\right)$ is a Jordan $\mathrm{C}^{*}$-algebra and

$$
|a|=\|a\| \quad(a \in \operatorname{Sym}(A)) .
$$

It is now enough to prove that

$$
|a|=\|a\| \quad(a \in A) .
$$

The first step is to prove that

$$
V_{1 \cdot \|}(a)=V_{\|\cdot\|}(a) \quad(a \in A) .
$$

To this end observe that, by [19; Theorem 7], $(A,|\cdot|)$ and $(A,\|\cdot\|)$ are V-algebras with the same hermitian elements. Hence if $a=h+i k(h, k \in H(A))$ we have

$$
\begin{aligned}
& \sup \operatorname{Re} V_{1} \cdot \mid(a)=\sup V_{1 \cdot \mid}(h), \\
& \sup \operatorname{Re} V_{\| \cdot{ }_{\|}}(a)=\sup V_{\|\cdot\|}(h) .
\end{aligned}
$$

But

$$
\sup V_{1 \cdot \mid}(h)=\sup V_{\|\cdot\|}(h)
$$

because both norms are equal on $H(A)$ and [3; Theorem 2.5] applies. Thus

$$
V_{1 \cdot 1}(a)=V_{\|\cdot\|}(a) \quad(a \in A) .
$$

Working now only with the original norm $\|\cdot\|$, let $T \in B L(A)$ and $a \in H(A)$. Then, as in the proof of Theorem 5,

$$
\left\|\exp \left(-i L_{a}\right) T(\exp (i a))\right\| \leqslant\|T\|
$$

and so the mapping $T \rightarrow \exp \left(-i L_{a}\right) T(\exp (i a))$ from $B L(A)$ into $A$ satisfies the conditions in Proposition 1. Thus

$$
\begin{aligned}
V_{\|\cdot\|}(T) & \supset \overline{\operatorname{co}} \bigcup\left\{V_{\|\cdot\|}\left(\exp \left(-i L_{a}\right) T(\exp (i a))\right): a \in H(A)\right\} \\
& =\overline{\operatorname{co}} \bigcup\left\{V_{1} \cdot\left(\exp \left(-i L_{a}\right) T(\exp (i a))\right): a \in H(A)\right\} .
\end{aligned}
$$

Applying Theorem 5, we have

and

$$
V_{\|} \cdot{ }_{\|}(T) \supset V_{\text {। }}(T) \quad(T \in B L(A)),
$$

$$
v_{\|\cdot\|}(T) \geqslant v_{|\cdot|}(T) \quad(T \in B L(A)) .
$$


By [9; Theorem 3], there is $k \in \mathbb{B}$ such that

$$
\|a\|=k|a| \quad(a \in A)
$$

and with $a=I$ we find that $k=1$.

Remark 7. In [9] it is assumed that

$$
v_{\|\cdot\|}(T)=v_{\mid} \cdot(T) . \quad(T \in B L(A)) .
$$

But really the proof only uses the weaker condition

$$
v_{\|\cdot\|}(T) \geqslant v_{|\cdot|}(T) \quad(T \in B L(A)) .
$$

If $A$ is an algebra we shall denote by $A^{+}$the algebra obtained from the vector space of $A$ with the product

$$
a \cdot b=\frac{1}{2}(a b+b a) .
$$

THEOREM 8. If $A$ is a $\mathrm{V}$-algebra the following statements are equivalent:

(a) $A$ is a noncommutative Jordan algebra;

(b) the natural involution of $A$ is multiplicative;

(c) the natural involution of $A$ is isometric.

Proof. (a) $\Leftrightarrow$ (b) by [10; Theorem IV.4.6]. (c) $\Rightarrow$ (b) is proved in [10; Theorem V.5.11]. It is enough to prove that (a) $\Rightarrow$ (c): $A^{+}$is a (commutative) Jordan Valgebra with the same natural involution as $A$. By Theorem 6 and [19; Lemma 4] this involution is isometric.

Theorems 6 and 8 include the classical Vidav-Palmer theorem ([11; Theorem 3.1]; see also [3; Theorem 6.9]) and also its generalization for alternative algebras ([10; Theorem IV.5.14]).

Corollary 9. If $A$ is an alternative $\mathrm{V}$-algebra, its natural involution $\left({ }^{*}\right)$ is multiplicative and $\left\|a^{*} a\right\|=\|a\|^{2}$ for all $a$ in $A$.

Proof. Multiplicativity of the involution is assured by Theorem 8 . To prove the star-property observe that $A^{+}$is a Jordan $\mathrm{V}$-algebra. So Theorem 6 applies and gives $\left\|U_{a}\left(a^{*}\right)\right\|=\|a\|^{3}$. Here $U_{a}\left(a^{*}\right)=a a^{*} a$. Hence $\|a\|^{2} \leqslant\left\|a^{*} a\right\|$.

Definition. Let $A$ and $B$ be noncomutative Jordan algebras. A J-homomorphism from $A$ into $B$ (respectively a J-derivation of $A$ ) will be an homomorphism from $A^{+}$ into $B^{+}$(respectively a derivation of $A^{+}$).

A linear mapping $F$ between two complex spaces with involution is said to be symmetric if $F\left(x^{*}\right)=(F(x))^{*}$.

The following theorem generalizes a result from Ylinen [18; Theorem 1.1]. The equivalence of assertion (a) and (b) of our theorem has been proved by Kadison ([7; see also [12]) in the associative case, and by Wright and Youngson [17] in the (commutative) Jordan case. 
Theorem 10. Let $A$ and $B$ be noncommutative Jordan V-algebras and $F$ a linear bijection from $A$ onto $B$. The following statements are equivalent:

(a) F is a symmetric J-isomorphism;

(b) $F$ is isometric and $F(I)=I$;

(c) $V(A, a)=V(B, F(a))$ for every $a$ in $A$.

Proof. (a) $\Rightarrow(\mathrm{b})$. This is proved by reduction to the commutative case, and using Theorem 6 and [15; Corollary 1.4].

(b) $\Rightarrow$ (c). This follows from Proposition 1 .

(c) $\Rightarrow$ (a). We may assume that $A$ and $B$ are commutative. Hence $H(A)$ and $H(B)$ are JB-algebras. As the norm of a hermitian element of a Jordan V-algebra is equal to its numerical radius, $F(I)=I$ and $F$ is an isometric linear bijection of $H(A)$ onto $H(B)$. So by $\left[17\right.$; Theorem 4] it follows that $F\left(a^{2}\right)=(F(a))^{2}$ for all $a \in H(A)$. Hence $F$ is a symmetric $\mathrm{J}$-isomorphism.

From the equivalence of assertions (a) and (b) of the above theorem, by arguments analogous to those in [14; Remark 3.5] or [20; Theorem 11], we obtain the following.

COROllary 11. Let $A$ be a noncommutative Jordan V-algebra and $T$ a mapping from $A$ into $A$. The following statements are equivalent:

(a) $T=L_{a}+i D$, where $a$ is a hermitian element of $A$ and $D$ is a symmetric $J$ derivation of $A$;

(b) $T$ is a hermitian element of $B L(A)$.

Remark 12. The above corollary generalizes the analogous results when $A$ is associative [14] or (commutative) Jordan [20]. Although in the associative case it is specified that $D$ is an authentic derivation of $A$, this is a consequence of the main result in [14; Theorem 3.3] which is obviously false even in the nonassociative alternative case. Noting that the complex octonions algebra can be structured as an V-algebra [10; Theorem IV.6.5] it can be seen that the above generalization is the best possible.

\section{Nonassociative $B^{*}$-algebras}

Definition. A nonassociative unital $\mathrm{B}^{*}$-algebra will be a unital complete normed complex algebra $A$ with an involution * satisfying

$$
I^{*}=I, \quad\left\|a^{*} a\right\|=\|a\|^{2} \quad(a \in A) .
$$

Corollary 9 actually shows that an alternative V-algebra with its (automatically multiplicative) natural involution is a nonassociative unital $\mathrm{B}^{*}$-algebra. In addition, as stated in Remark 12, there are alternative $\mathrm{V}$-algebras which are not associative.

Our purpose in this section is to show (answering affirmatively the question posed in [10]) that, conversely, nonassociative unital $\mathrm{B}^{*}$-algebras are alternative $\mathrm{V}$ algebras. We begin with a basic but elementary lemma. 
LeMma 13. Let $A$ be a unital complete normed algebra and let $\sum(1 / n !) r^{n} F_{n}$ be a power series with coefficients $F_{n}$ in $B L(A)$. Assume the existence of a positive number $k$ such that the series converges and that, if $G_{r}$ denotes the sum, then $G_{r}$ is an automorphism of $A$, for $|r|<k(r \in \mathbb{R})$. Then

(a) $F_{n}(a b)=\sum_{i=0}^{n}\left(\begin{array}{l}n \\ i\end{array}\right) F_{i}(a) F_{n-i}(b)$ for all $a$ and $b$ in $A$,

(b) in particular if $F_{0}=I$ and $F_{1}=0$ then $F_{2}$ is a derivation of $A$.

Proof. We have $G_{r}(a b)=G_{r}(a) G_{r}(b)(|r|<k ; a, b \in A)$ and on writing the second member as a Cauchy product of the corresponding series and identifying coefficients we obtain (a); (b) is clear.

THEOREM 14. Every nonassociative unital $B^{*}$-algebra is an alternative $V$-algebra whose natural involution agrees with the original one (which is in consequence multiplicative by Corollary 9).

Proof. Let $A$ be a unital $\mathrm{B}^{*}$-algebra. Then clearly (using an argument analogous to [3; Example 5.3]), Sym $(A) \subset H(A)$. So $A$ is a V-algebra whose natural involution coincides with the original one.

The equality $\left\|a^{*}\right\|=\|a\|(a \in A)$, which is clear from the definition of unital $\mathrm{B}^{*}$ algebras, implies in view of Theorem 8 that $A$ is a noncommutative Jordan algebra with multiplicative involution (up to now everything is in [10]). Let $h \in H(A)$. If $r \in \mathbb{R}$, we define

$$
G_{r}=L_{\exp (i r h)} \exp \left(-i r L_{h}\right)
$$

We want to prove that $V\left(G_{r}(a)\right)=V(a)(a \in A)$.

If $s \in \mathbb{R}$, then

$$
\begin{aligned}
\|I+s a\|^{2} & =\left\|\exp (-i r h)+s \exp \left(-i r L_{h}\right)(a)\right\|^{2} \\
& =\left\|\left[\exp (i r h)+s\left(\exp \left(-i r L_{h}\right)(a)\right)^{*}\right]\left[\exp (-i r h)+s \exp \left(-i r L_{h}\right)(a)\right]\right\| \\
& =\left\|I+s\left(G_{r}(a)+\left(G_{r}(a)\right)^{*}\right)+s^{2} \ldots\right\|
\end{aligned}
$$

(the first equality is true because $\exp \left(-i r L_{h}\right)$ is an isometry, the second one by definition of unital $\mathrm{B}^{*}$-algebras and the third one is clear). 2.5]

Computing the first and second right derivatives at $s=0$, we have [3; Theorem

$$
2 \sup \operatorname{Re} V(a)=\sup V\left(G_{r}(a)+\left(G_{r}(a)\right)^{*}\right) .
$$

Hence

$$
\sup \operatorname{Re} V(a)=\sup \operatorname{Re} V\left(G_{r}(a)\right) .
$$

Thus we have proved that

$$
V(a)=V\left(G_{r}(a)\right) \quad(a \in A, \quad r \in \mathbb{R}) .
$$


As $G_{0}=I$ and $r \rightarrow G_{r}$ is continuous, we may assume that there exists a positive number $k$ such that, for $|r|<k, G_{r}$ is invertible in $B L(A)$. Thus, for $|r|<k, G_{r}$ is a linear bijection satisfying $V\left(G_{r}(a)\right)=V(a)$. By Theorem $10, G_{r}$ is a symmetric $\mathrm{J}$ automorphism.

If $\sum(1 / n !) r^{n} F_{n}$ is the power series development of $G_{r}$, we have $F_{0}=I, \quad F_{1}=0$, $F_{2}=L_{h}^{2}-L_{h^{2}}$. By the previous lemma, $L_{h}^{2}-L_{h^{2}}$ is a symmetric J-derivation of $A$. By Corollary 11 we have $L_{h}^{2}-L_{h^{2}} \in i H(B L(A))$ and by [3; Theorem 2.6], $\operatorname{sp}\left(L_{h}^{2}-L_{h^{2}}\right) \subset i \mathbb{R}$. We have also $\operatorname{sp}\left(L_{h}\right) \subset \mathbb{P}$ and $\operatorname{sp}\left(L_{h^{2}}\right) \subset \mathbb{R}$. And as $L_{h}$ and $L_{h^{2}}$ commute

$$
\operatorname{sp}\left(L_{h}^{2}-L_{h^{2}}\right) \subset \operatorname{sp}\left(L_{h}^{2}\right)-\operatorname{sp}\left(L_{h^{2}}\right)=\left(\operatorname{sp}\left(L_{h}\right)\right)^{2}-s p\left(L_{h^{2}}\right) \subset \mathbb{R} .
$$

So $\operatorname{sp}\left(L_{h}^{2}-L_{h^{2}}\right)=\{0\}$. Then by the Sinclair theorem (see [4; Theorem 26.2]) or, alternatively, by the Vidav lemma [3; Corollary 5.11] and Bohnenblust-Karlin theorem [3; Theorem 4.1] we have $L_{h}^{2}-L_{h^{2}}=0$. Since for any element $b$ of a noncommutative Jordan algebra the identity $R_{b}^{2}-R_{b^{2}}=L_{b}^{2}-L_{b^{2}}$ holds (see [13; p. 142]), we have also $R_{h}^{2}-R_{h^{2}}=0$, and $A$ is alternative.

As in [3; Theorem 7.7], an easy consequence of the previous theorem and Corollary 9 is the following.

COROLlary 15. If $A$ is a nonassociative unital $B^{*}$-algebra and $M$ is a proper closed two-sided ideal of $A$, then $M$ is selfadjoint and $A / M$ with the quotient involution is a nonassociative $B^{*}$-algebra.

If $a$ is an element of an algebra $A$, then $L_{a}^{+}$denotes the mapping $b \rightarrow b$. $a$ from $A$ into $A$, where " ." is the product of $A^{+}$.

THEOREM 16. Let $A$ be a unital complete normed complex algebra with an involution * satisfying

$$
\left(a^{*}\right)^{2}=\left(a^{2}\right)^{*}, \quad\left\|a^{*} a\right\|=\left\|a^{*}\right\|\|a\|
$$

for all $a$ in $A$. Then $A$ is a nonassociative $B^{*}$-algebra.

Proof. If $T \in L(A)$ (the associative algebra of not necessarily bounded linear maps from $A$ into $A$ ), we define $T^{*} \in L(A)$ by $T^{*}(a)=\left(T\left(a^{*}\right)\right)^{*}$ for all $a$ in $A$. Then $T \rightarrow T^{*}$ is an involution on $L(A)$ satisfying $\left(T_{1} T_{2}\right)^{*}=T_{1}^{*} T_{2}^{*}$. Hence $\operatorname{sp}\left(L(A), T^{*}\right)=\overline{\operatorname{sp}(L(A), T)}$ for all $T \in L(A)$. The assumption $\left(a^{*}\right)^{2}=\left(a^{2}\right)^{*}$ for all $a \in A$ (equivalent to the multiplicativity of * in $\left.A^{+}\right)$is also equivalent to $\left(L_{a}^{+}\right)^{*}=L_{a}$. for all $a \in A$. Since $B L(A)$ is a full subalgebra of $L(A)$

$$
\operatorname{sp}\left(B L(A), L_{a}^{+}\right)=\overline{\operatorname{sp}\left(B L(A), L_{a}^{+}\right)} .
$$

As a consequence, if $h$ is an hermitian element of $A$,

$$
\operatorname{sp}\left(L_{h}^{+}\right)=\operatorname{sp}\left(L_{h}^{+}\right) \subset \mathbb{R} .
$$

Let $a \in \operatorname{Sym}(A)$. As in the proof of [3; Theorem 7.2], $a=h+i r I$ with $h \in H(A)$ 
and $r \in \mathbb{R}$. Then $L_{a}^{+}=L_{h}^{+}+$irI and $L_{a}^{+}=L_{h^{+}}^{+}-i r I$. Hence $\operatorname{sp}\left(L_{a}^{+}\right) \subset \mathbb{R}+i r$ and $\operatorname{sp}\left(L_{a}^{+}\right) \subset \mathbb{R}-i r$, which implies that $r=0$.

We have shown that $\operatorname{Sym}(A) \subset H(A)$. Hence $A$ (and also $A^{+}$) is a V-algebra whose natural involution agrees with the original one, which is multiplicative in $A^{+}$ by assumption. Then by Theorem $8,{ }^{*}$ is isometric. Hence $\left\|a^{*} a\right\|=\|a\|^{2}$ for all $a \in A$ and $A$ is a nonassociative $\mathrm{B}^{*}$-algebra, as required.

Remark 17. As asserted in Theorem 14, the assumption of multiplicativity of the involution in the Gelfand-Naimark axioms is too strong and may be reduced to the apparently weaker $I^{*}=I$. This is because the proof that every unital associative $\mathrm{B}^{*}$. algebra is a V-algebra only uses this last fact, the multiplicativity of the natural involution of associative V-algebras being automatic (a result of Palmer [11], contained in Theorem 8).

In the same way, in the definition of Jordan $\mathrm{C}^{*}$-algebras, the multiplicativity of involution may be replaced by $I^{*}=I$.

\section{The second conjugate space of a V-algebra}

Definitions and notation. If $X$ is a normed space and $F$ is a continuous bilinear mapping from $X \times X$ into $X$, then $F^{T}\left(: X^{\prime \prime} \times X^{\prime \prime} \rightarrow X^{\prime \prime}\right)$ will denote the third Arens transpose of $F$ [2]. Denoting by $F^{R}$ the mapping $(x, y) \rightarrow F(y, x)$ from $X \times X$ into $X$, we recall that $F$ is called Arens-regular if $F^{T R}=F^{R T}$. The second conjugate space $A^{\prime \prime}$ of a unital normed algebra $A$ is considered as a new unital normed algebra with the product $F^{T}$ (where $F$ stands for the product in $A$ ); $A$ will be said to be Arens-regular if its product is Arens-regular.

Let $X$ be a complex normed space with continuous involution *. For $f \in X^{\prime}$ we define $f^{*}$ by $f^{*}(x)=\overline{f\left(x^{*}\right)}$ for all $x \in X$. Then $f \rightarrow f^{*}$ is a continuous involution on $X^{\prime}$ which we shall call the "transpose" of the one on $X$ and which is isometric if the given one is isometric.

If $F$ is a continuous bilinear mapping from $X \times X$ into $X$, then $F^{*}$ will denote the mapping $(x, y) \rightarrow\left(F\left(x^{*}, y^{*}\right)\right)^{*}$ from $X \times X$ into $X$ and, analogously, $F^{T *}$ will denote the mapping $(u, v) \rightarrow\left(F^{T}\left(u^{*}, v^{*}\right)\right)^{*}$ from $X^{\prime \prime} \times X^{\prime \prime}$ into $X^{\prime \prime}$ where, now, the involution $*$ is the second transpose of the given one.

With this notation we have the following lemma, whose proof is routine.

Lemma 18. $\quad F^{T *}=F^{* T}$.

Corollary 19. Let $A$ be a unital normed algebra with multiplicative continuous involution *. Then the second transposed involution is multiplicative on $A^{\prime \prime}$ if and only if $A$ is Arens-regular.

Proof. Let $F$ be the product of $A$. The multiplicativity of * is written $F^{*}=F^{R}$. By Lemma $18, F^{T *}=F^{R T}$ and so $F^{R T}=F^{T R}$ (the Arens-regularity of $A$ ) is equivalent to $F^{T *}=F^{T R}$ (the multiplicativity of * in $A^{\prime \prime}$ ).

If $A$ is an Arens-regular unital normed algebra, the product of $A^{\prime \prime}$ is separately weak-star-continuous in its variables. This fact and the weak-star-density of $A$ in $A^{\prime \prime}$ lead to the next lemma. 
Lemma 20. If $A$ is an Arens-regular unital normed algebra, then $A^{\prime \prime}$ satisfies any multilinear identity satisfied by $A$.

Proposition 21. Let $A$ be c : :nital normed algebra and $G \in A^{\prime \prime}$. Then

$$
V\left(A^{\prime \prime}, G\right)=\left\{G(f): f \in A^{\prime},\|f\|=f(I)=1\right\}^{-}
$$

(where - denotes topological closure).

Proof. An analysis of the proof for the analogous associative result [3; Theorem 12.2] shows that associativity is not used.

Proposition 22. If $A$ is a V-algebra, then $A^{\prime \prime}$ is a V-algebra with natural involution the second transpose of the natural one of $A$.

Proof. If * denotes indiscriminately the natural involution of $A$ and the first and second transposed ones, it is enough to prove that ${ }^{*}-\operatorname{Sym}\left(A^{\prime \prime}\right) \subset H\left(A^{\prime \prime}\right)$. Let $G \in^{*}-\operatorname{Sym}\left(A^{\prime \prime}\right)$ and $f \in A^{\prime}$ with $\|f\|=f(I)=1$. Then clearly $f \in{ }^{*}-\operatorname{Sym}\left(A^{\prime}\right)$. So $G(f) \in \mathbb{R}$. Proposition 21 completes the proof.

TheOrem 23. Let $A$ be a noncommutative Jordan V-algebra. Then $A^{\prime \prime}$ is a V-algebra satisfying any multilinear identity satisfied by $A$. In particular $A^{\prime \prime}$ is a noncommutative Jordan V-algebra.

Proof. By Theorem 8 the natural involution of $A$ is multiplicative and isometric. By Proposition 22, $A^{\prime \prime}$ is a V-algebra whose natural involution (the second transpose) will be isometric too. Again by Theorem 8 the natural involution of $A^{\prime \prime}$ is multiplicative. By Corollary 19, $A$ is Arens-regular and Lemma 20 completes the proof.

Corollary 24. The bidual of a Jordan $\mathrm{C}^{*}$-algebra $A$, with involution the second transpose of the involution of $A$, is a new Jordan $\mathrm{C}^{*}$-algebra.

Proof. By [19; Theorem 7], $A$ is a Jordan V-algebra whose natural involution is the original one. Now apply Theorem 23, Proposition 22 and Theorem 6.

COROllary 25. The bidual of a nonassociative unital B*-algebra, with the second transpose involution, is a nonassociative unital $\mathrm{B}^{*}$-algebra.

Proof. Use Theorem 14, Theorem 23, Proposition 22 and Corollary 9.

4. The numerical index of a noncommutative Jordan V-algebra

Definition. The numerical index, $n(A)$, of a unital normed algebra $A$ is defined by the formula

$$
n(A)=\inf \{v(a): a \in A,\|a\|=1\} .
$$


TheOREM 26. The numerical index of a noncommutative Jordan V-algebra $A$ is 1 or $\frac{1}{2}$ depending on whether or not $A$ is associative and commutative.

The proof of this theorem needs some preliminary results. Some of them (Lemmas 27 and 28 and Proposition 29) are essentially part of the classical theory of Banach algebras but it may be that they are not well-known.

Lemma 27. Let $a$ and $b$ be elements of an (associative) Banach algebra $A$. Then

$$
r(a+b) \leqslant \max (\|a\|,\|b\|)+\sqrt{\|a b\|}
$$

(where $r$ is the spectral radius).

Proof. Obviously we may suppose $A$ to be complex and unital. Then the assertion is equivalent to

$$
|c| \leqslant \max (\|a\|,\|b\|)+\sqrt{\|a b\|}
$$

for all $c \in \operatorname{sp}(a+b)$. Since this inequality is evident if $|c| \leqslant \max (\|a\|,\|b\|)$, let us suppose that $|c|>\max (\|a\|,\|b\|)$. Then $c I-a$ and $c I-b$ are invertible and

$$
\left\|(c I-a)^{-1}\right\| \leqslant(|c|-\|a\|)^{-1},\left\|(c I-b)^{-1}\right\| \leqslant(|c|-\|b\|)^{-1} .
$$

Hence

$$
\left\|((c I-a)(c I-b))^{-1}\right\| \leqslant(|c|-\max (\|a\|, \| b||))^{-2} .
$$

Since $\quad(c I-a)(c I-b)-c(c I-(a+b))=a b, \quad$ and $\quad c(c I-(a+b)) \quad$ is singular $(c \in \operatorname{sp}(a+b) !)$, the distance from $c(c I-(a+b))$ to $(c I-a)(c I-b)$, which equals $\|a b\|$, must be equal to or greater than $\left\|((c I-a)(c I-b))^{-1}\right\| \|^{-1}$; this and the last inequality complete the proof.

Lemma 28. Let $A$ be a Jordan $\mathrm{C}^{*}$-algebra. Then

$$
\left\|a^{*} . a\right\| \leqslant\left(\frac{1}{2}\right)\left(\|a\|^{2}+\sqrt{\left\|U_{a^{*}}\left(a^{2}\right)\right\|}\right)
$$

for all $a$ in $A$.

Proof. The reduction to $A=B^{+}$, with $B$ an associative $\mathrm{B}^{*}$-algebra, is a consequence of [15; Corollary 2.2] (the associative product of $B$ will be noted by juxtaposition while “." will denote the product of $B^{+}$). Then, by Lemma 27 ,

$$
\left\|b^{*} b+b b^{*}\right\| \leqslant\|b\|^{2}+\sqrt{\left\|b^{*} b^{2} b^{*}\right\|}
$$

for all $b \in B$, which, with notation of $B^{+}$, is

$$
\left\|b^{*} . b\right\| \leqslant\left(\frac{1}{2}\right)\left(\|b\|^{2}+\sqrt{\left\|U_{b^{*}}\left(b^{2}\right)\right\|}\right) .
$$


Proposition 29. Let $A$ be a noncommutative Jordan V-algebra. Then

$$
\left(\frac{1}{2}\right)\|a\| \leqslant v(a) \leqslant\left(\frac{1}{2}\right)\left(\|a\|+\sqrt{\left\|a^{2}\right\|}\right)
$$

for all $a$ in $A$.

Proof. The first inequality is clear. For the second one, by multiplication by a unimodular number which does not affect the result, we may suppose that $v(a)=\max \operatorname{Re} V(a)$. Let $h=\left(\frac{1}{2}\right)\left(a+a^{*}\right)$. Then

$$
\begin{aligned}
(v(a))^{2} \leqslant\|h\|^{2}=\left\|h^{2}\right\| & =(1 / 4)\left\|a^{2}+a^{* 2}+a^{*} a+a a^{*}\right\| \\
& \leqslant\left(\frac{1}{2}\right)\left(\left\|a^{2}\right\|+\left\|a^{*} \cdot a\right\|\right),
\end{aligned}
$$

where . denotes the product of $A^{+}$which, by Theorem 6, is a Jordan C*-algebra. A consequence of Lemma 28 is

Hence

$$
\left\|a^{*} . a\right\| \leqslant\left(\frac{1}{2}\right)\left(\|a\|^{2}+\|a\| \sqrt{\left\|a^{2}\right\|}\right) .
$$

$$
\begin{aligned}
(v(a))^{2} & \leqslant(1 / 4)\left(2\left\|a^{2}\right\|+\|a\| \sqrt{\left\|a^{2}\right\|}+\|a\|^{2}\right) \\
& =(1 / 4)\left(\left\|a^{2}\right\|+\sqrt{\left\|a^{2}\right\|} \sqrt{\left\|a^{2}\right\|}+\|a\| \sqrt{\left\|a^{2}\right\|}+\|a\|^{2}\right) \\
& \leqslant(1 / 4)\left(\left\|a^{2}\right\|+\|a\| \sqrt{\left\|a^{2}\right\|}+\|a\| \sqrt{\left\|a^{2}\right\|}+\|a\|^{2}\right) \\
& =(1 / 4)\left(\sqrt{\left\|a^{2}\right\|}+\|a\|\right)^{2},
\end{aligned}
$$

and this completes the proof.

Lemma 30. Let $A$ be a unital normed noncommutative Jordan algebra. The following statements are equivalent:

(a) there exists $k>0$ such that $\|a\| \leqslant k r(a)$ for all $a$ in $A$;

(b) there exists $r>0$ such that $\|a\|^{2} \leqslant r\left\|a^{2}\right\|$ for all $a$ in $A$.

Proof. The algebra $A$ is power-associative and hence an associative argument is valid.

Proposition 31. If the equivalent statements of Lemma 30 are verified and $A$ is complete and complex, then $A$ is associative and commutative.

Proof. Let $D$ be a continuous J-derivation of $A$ and let $z \in \mathbb{C}$. Then $\exp (z D)$ is a $\mathrm{J}$-automorphism and, using (a),

$$
\|\exp (z D)(a)\| \leqslant k r(\exp (z D)(a))=k r(a) \leqslant k\|a\|
$$

for all $a$ in $A$. Hence $\|\exp (z D)\| \leqslant k$. By Liouville's theorem, $D=0$. If $a, b \in A$, then $L_{a}-R_{a}$ and $\left[L_{a}, R_{b}\right]$ are continuous J-derivations of $A$ (see [13; p. 146]); hence both equal zero. So $A$ is associative and commutative. 
Proof of Theorem 26. Let $A$ be the noncommutative Jordan V-algebra under consideration. By the first inequality in Proposition $29, n(A) \geqslant \frac{1}{2}$. So, to prove the theorem, it is enough to show that if $n(A)>\frac{1}{2}$ then $A$ is associative and commutative and then it is known that $n(A)=1$.

If $n(A)>\frac{1}{2}$, by the second inequality of Proposition 29

$$
n(A)\|a\| \leqslant\left(\frac{1}{2}\right)\left(\|a\|+\sqrt{\left\|a^{2}\right\|}\right) \Leftrightarrow(2 n(A)-1)^{2}\|a\|^{2} \leqslant\left\|a^{2}\right\|
$$

for all $a$ in $A$. Then, by Proposition 31, $A$ is associative and commutative.

Corollary 32. If $A$ is a $V$-algebra with $n(A)=1$, then $A$ is associative and commutative.

Proof. For all $a$ in $A, n(A)=1 \Leftrightarrow v(a)=\|a\|$. But, if * denotes the natural involution of $A$, the equality $v\left(a^{*}\right)=v(a)$ is clear. Then * is isometric. By Theorem 8 , $A$ is a noncommutative Jordan algebra. Theorem 26 completes the proof.

The following corollary is a generalization of a result from Huruya [5].

Corollary 33. Let $A$ be a noncommutative Jordan V-algebra. Then the numerical index of $B L(A)$ is 1 or $\frac{1}{2}$ depending on whether or not $A$ is associative and commutative.

Proof. Clearly $A$ may be supposed to be commutative and so, applying consecutively Theorems 6 and 5 , we obtain

$$
V(T)=\overline{\operatorname{co}} \bigcup\left\{V\left(\exp \left(-i L_{a}\right) T(\exp (i a))\right): a \in H(A)\right\}
$$

which implies

$$
\begin{aligned}
v(T) & =\sup \left\{v\left(\exp \left(-i L_{a}\right) T(\exp (i a))\right): a \in H(A)\right\} \\
& \geqslant n(A) \sup \left\{\left\|\exp \left(-i L_{a}\right) T(\exp (i a))\right\|: a \in H(A)\right\}=n(A)\|T\|
\end{aligned}
$$

for all $T$ in $B L(A)$. Thus $n(B L(A)) \geqslant n(A)$ and, since the opposite inequality is valid for any unital normed algebra $A$, we have $n(B L(A))=n(A)$. Theorem 26 completes the proof.

\section{References}

1. E. M. Alfsen, F. W. Shultz and E. Størmer, "A Gelfand-Neumark theorem for Jordan algebras", Adv. in Math., 28 (1978), 11-56.

2. R. Arens, "The adjoint of a bilinear operation", Proc. Amer. Math. Soc., 2 (1951), 839-848.

3. F. F. Bonsall and J. Duncan, Numerical ranges of operators on normed spaces and of elements of normed algebras, London Math. Soc. Lecture Note Series 2 (Cambridge, 1971).

4. F. F. Bonsall and J. Duncan, Numerical ranges II, London Math. Soc. Lecture Note Series 10 (Cambridge, 1973).

5. T. Huruya, "The normed space numerical index of C*-algebras", Proc. Amer. Math. Soc., 63 (1977), 289-290. 
6. N. Jacobson, "Structure and representation of Jordan algebras", Amer. Math. Soc. Colloquium Publications 39 (American Mathematical Society, Providence, 1968).

7. R. V. Kadison, "Isometries of operator algebras", Ann. of Math., 54 (1951), 325-338.

8. J. Martinez, "Sobre algebras de Jordan normadas completas", Tesis Doctoral, Secretariado de Publicaciones de la Universidad de Granada, Granada, 1977.

9. C. M. McGregor, "Operator norms determined by their numerical ranges", Proc. Edinburgh Math. Soc. (2), 17 (1971), 249-255.

10. A. Mojtar, "Bases para una teoría de las algebras no asociativas normadas", Tesis Doctoral, Secretariado de Publicaciones de la Universidad de Granada, Granada, 1978.

11. T. W. Palmer, "Characterization of C*-algebras II", Trans. Amer. Math. Soc., 148 (1970), 577-588.

12. A. L. T. Paterson, "Isometries between B*-algebras", Proc. Amer. Math. Soc., 22 (1970), 570-572.

13. R. D. Schafer, An introduction to nonassociative algebras (Academic Press, New York-London, 1966).

14. A. M. Sinclair, "Jordan homomorphisms and derivations on semisimple Banach algebras", Proc. Amer. Math. Soc., 24 (1970), 209-214.

15. J. D. M. Wright, “Jordan C*-algebras", Michigan Math. J., 24 (1977), 291-302.

16. J. D. M. Wright and M. A. Youngson, "A Russo Dye theorem for Jordan C*-algebras", Functional analysis: surveys and recent result (North Holland, Amsterdam, 1977), pp. 279-282.

17. J. D. M. Wright and M. A. Youngson, "On isometries of Jordan algebras", J. London Math. Soc. (2), 17 (1978), 339-344.

18. K. Ylinen, "Vector space isomorphism of C*-algebras", Studia Math., 46 (1973), 31-34.

19. M. A. Youngson, "A Vidav theorem for Banach Jordan algebras", Math. Proc. Cambridge Philos. Soc., 84 (1978), 263-272.

20. M. A. Youngson, "Hermitian operators on Banach Jordan algebras", Proc. Edinburgh Math. Soc. (2), 22 (1979), 93-104.

\author{
Universidad de Granada, \\ Facultad de Ciencias, \\ Departamento de Teoría de Funciones, \\ Granada, \\ Spain.
}

